\title{
Suicide Risk in Youth and Young Adults with Type 1 Diabetes: a Review of the Literature and Clinical Recommendations for Prevention
}

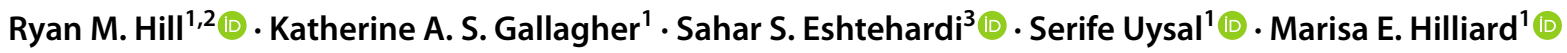

Accepted: 28 June 2021 / Published online: 13 December 2021

(c) The Author(s), under exclusive licence to Springer Science+Business Media, LLC, part of Springer Nature 2021

\begin{abstract}
Purpose of Review The manuscript reviews the extant literature on suicide-related thoughts and behaviors among youth and young adults with pediatric diabetes. This evidence is presented within the context of current theories of the etiology of suicidal behavior to highlight how diabetes may contribute to suicide risk, and to support providers in understanding the interplay between pediatric diabetes and suicide risk. The manuscript also reviews evidence-based approaches to suicide prevention suitable for use in pediatric healthcare settings, with suggestions for their application to this unique population. Recent Findings Several recent studies identify heightened rates of suicidal ideation, suicide attempts, and suicide among youth and young adults with pediatric diabetes, as compared with their peers without diabetes. Evidence-based suicide prevention approaches frequently emphasize the importance of reducing suicidal youths' access to potentially lethal means for suicidal behavior. This approach may require special considerations for youth with pediatric diabetes, due to their need to carry sufficient quantities of insulin and the dangers of inaccurate insulin dosing and/or overdose. Suggestions for suicide prevention for this population include risk screening as part of routine diabetes care, early prevention, education for youth and families, and provider awareness of risk factors, warning signs, and implications for diabetes care.

Summary Youth and young adults with diabetes reported elevated rates of suicide-related behaviors as compared with their peers without diabetes. Existing suicide prevention approaches may require substantial adaptation for use with youth and young adults with diabetes. Further research is needed to examine how to best prevent suicidal behaviors among this population.
\end{abstract}

Keywords Pediatrics $\cdot$ Diabetes $\cdot$ Type 1 diabetes $\cdot$ Suicide $\cdot$ Suicide attempt

Suicide-related behaviors are a serious public health concern. Suicide is the 2 nd leading cause of death among youth and young adults, ages $10-34$, in the USA and accounted for more than 14,000 deaths among children and adolescents between 2010 and 2019 [1]. During that same period, the rate of suicide among 10-17 year olds increased

This article is part of the Topical Collection on Psychosocial Aspects

Marisa E. Hilliard

marisa.hilliard@bcm.edu

1 Department of Pediatrics, Baylor College of Medicine, Texas Children's Hospital, Houston, TX, USA

2 College for Health, Community and Policy, University of Texas at San Antonio, San Antonio, TX, USA

3 Department of Psychological, Health, \& Learning Sciences, University of Houston, Houston, TX, USA approximately $65 \%$, from 2.98 per 100,000 in 2010 to 4.91 per 100,000 in 2019 [1]. In addition, data from the nationally representative Youth Risk Behavior Survey indicate that $18.8 \%$ of high school students have seriously considered suicide in the previous 12 months and $8.9 \%$ made a suicide attempt over that same period [2]. Rates of adolescent suicidal ideation and suicide attempts also appear to be more elevated during the COVID-19 pandemic, as compared with pre-COVID-19 rates [3].

Data indicate that suicidal ideation and attempts are elevated among youth and young adults with chronic health conditions [4, 5]. In particular, suicide attempts and other behavioral health diagnoses appear to be elevated among youth with type 1 diabetes (T1D) [6, 7]. Furthermore, behavioral health concerns in youth with T1D are associated with higher HbAlc levels, less engagement in self-management behaviors such as blood glucose monitoring, and lower quality of life [8-11]. As evidence of increased behavioral 
health concerns among youth with T1D has emerged, there have been increased efforts to implement routine screening for psychosocial issues, including depressive symptoms, in pediatric diabetes clinics [12-19]. Suicide risk screening is also becoming more common in healthcare settings, based on recommendations from the Joint Commission and suicide prevention organizations [20-24]. As a consequence, clinic staff and providers are increasingly in a position of needing to understand and respond to behavioral health concerns, including suicide risk, quickly and effectively in a range of healthcare settings.

\section{Increased Risk of Suicide-Related Behaviors Among Youth and Young Adults with T1D}

With respect to suicidal ideation, a nationally representative study of Canadian adolescents and adults reported that individuals with T1D had a higher lifetime prevalence of suicidal ideation (15.0\%) as compared with people without T1D (9.4\%) [25]. Similarly, Goldston and colleagues [26, 27] reported an elevated rate of suicidal ideation among children and adolescents with T1D, with $29.5 \%$ reporting suicidal ideation within 2-3 weeks of their diagnosis and $46 \%$ reporting suicidal ideation over a 12-year follow-up period. With respect to suicide attempts, in a Swedish population-based case cohort study, children and adolescents with T1D were 1.7 times more likely to make a suicide attempt, as compared with matched controls [6]. Young adults who have been hospitalized for diabetic ketoacidosis are also at increased risk for suicide attempt ( $2.2 \%$ vs. $0.5 \%$ among those not hospitalized for diabetic ketoacidosis, in the 12 months following hospitalization) [28•].

With respect to suicide deaths, a meta-analysis of more than 50 studies reported that individuals with T1D have a risk of suicide 2.25 times that of those without T1D [29•]. A systematic review published in 2014 concluded that patients with T1D were at elevated risk for suicide-related behaviors, though the extent of that increased risk was unclear based on the existing evidence [30]. In that review, the rate of suicide deaths among adults with T1D was approximately 43.1 per 100,000 people [30-36], as compared with a globalized suicide rate of approximately 10.5 per 100,000 [37]. Among youth, the suicide rate was approximately double that, at 87.2 per 100,000 people [30,38-43]. However, results have not been uniform, with perhaps the largest study to date, an evaluation of over 28,000 youth with T1D in 12 European nations, reporting no significant elevations in suicide deaths as compared with controls without T1D [39]. The reason for these discrepant findings is unclear and further populationlevel studies are needed. Furthermore, youth with T1D are also at increased risk for a range of psychiatric disorders, including mood, anxiety, and eating disorders, substance misuse, and other behavioral disorders [6, 8], each of which have been associated with suicide risk among youth [44, 45]. Youth with T1D also experience elevated rates of family conflict [46], stress [47], social and peer pressure [48], and school difficulties [49], all of which have been associated with suicide risk in youth [50-56]. Taken together, these studies indicate that youth with T1D are at elevated risk for suicide-related behaviors, including suicidal ideation, suicide attempts, and death by suicide, as well as a range of factors associated with suicide-related behaviors.

\section{Theoretical Frameworks for Understanding Suicide-Related Behaviors in Youth and Young Adults with T1D}

Given the elevated rate of suicide-related behaviors among youth with T1D, it is important that medical and behavioral health providers have a conceptual understanding of the development of suicide risk among children and adolescents. Understanding the etiology of suicide-related behaviors and recognizing warning signs can aid providers in identifying at-risk individuals and directing them to appropriate resources or interventions. Several theories of the etiology of suicide risk have received substantial empirical support in recent years [57-60]. These theories of suicidal behavior share a common approach to understanding suicide within "ideation-to-action" frameworks [58]. Ideation-to-action frameworks separate the development of suicide risk into two distinct causal processes, the first are processes that lead to suicidal ideation (e.g., as a result of unmet psychological needs) and the second are processes that enable individuals with suicidal ideation to engage in suicidal action (i.e., serious suicide attempts or death by suicide) [58].

The interpersonal-psychological theory of suicide (IPTS) $[57,59]$ has received considerable attention and empirical support in recent years [61-63]. The IPTS proposes that the desire for death (i.e., suicidal ideation) results from the joint presence of two proximal risk factors: thwarted belongingness and perceived burdensomeness [59]. Thwarted belongingness is a perception of social isolation, loneliness, or disconnection from others [57] and has been operationalized as chronically strained relationships with family, close friends, and romantic partners, as well as a lack of social support or relationships, all of which have been associated with more severe suicidal behaviors in adolescence [64, 65]. Perceived burdensomeness is primarily conceptualized as the belief that one has become a burden on others or that one's existence is a drain on the resources of others [57]. In youth, perceived burdensomeness frequently manifests as a perception of being a financial drain on parents and family, of being a disappointment to others, or of letting others down [66]. The IPTS also proposes that the shift from 
suicidal ideation to serious suicidal behavior occurs in the presence of the acquired capability for suicide. The acquired capability refers to an individual's ability to overcome the fear of pain and death, a necessary component to engaging in serious suicidal behavior [57]. Required capacity results from repeated exposure to painful or provocative events, essentially building up tolerance for pain, discomfort, and self-harm [57].

Another ideation-to-action model, the 3-step theory of suicide (3ST) proposes that suicidal ideation begins with the experience of pain (either physical or emotional), coupled with a sense of hopelessness about the future [58]. Suicidal ideation becomes more intense when an individual experiences low connectedness, similar to the concept of thwarted belongingness in the IPTS. Finally, the 3ST proposes that progression from suicidal ideation to suicidal behavior occurs when an individual has the capacity to engage in suicidal behavior. The 3ST model provides a broad definition of capacity that includes acquired elements (i.e., the acquired capability from the IPTS), dispositional elements (e.g., pain sensitivity), and practical elements (e.g., access to firearms, medications).

Specific aspects of living with and managing T1D have implications for how these theories translate to risk in youth and young adults with T1D. Perhaps most notably, youth with T1D have a high degree of access to potentially lethal means of suicide, via their access to insulin, which has been reported as a method of suicide attempt among youth with T1D [67]. However, removing access to such medication raises additional concerns, as youth with T1D might also skip medication doses, with the intent to trigger diabetic ketoacidosis, a potentially lethal outcome. The medications and supplies for T1D management (e.g., insulin, glucagon, management devices) are also expensive [68, 69], and completing the many complex tasks of daily T1D management requires substantial accommodation to youth and family routines [70-72]. Together, these basic requirements of T1D management may lead youth to perceive themselves as a burden on family time and financial well-being. In a study of youth and young adults with T1D who screened positive for suicide risk, $65 \%$ reported that they felt like a burden on their families, with T1D being a common reason for this perception, and 47\% reported a low fear of death [73].

Youth with T1D also frequently report poor social support and low connectedness, particularly youth from lower socioeconomic backgrounds [74, 75]. Youth with T1D may thus experience elevations in both perceived burdensomeness and thwarted belongingness. Further, management of T1D often involves repeated engagement in potentially painful self-management tasks (e.g., frequent blood glucose checks and insulin injections, site changes for continuous glucose monitors and insulin pumps). This repeated exposure to mild pain and the sight of blood may contribute to the development of the acquired capability for engaging in suicidal behaviors. Repeated hypoglycemic experiences may also serve as a means for building tolerance to the discomfort associated with hypoglycemia, resulting in an acquired capability for insulin overdose, a high-risk method of suicide for people with T1D. Finally, consistent with the 3ST model, the knowledge that T1D is a chronic, lifelong condition, paired with difficulties in diabetes self-management, may lead youth with T1D to experience a sense of hopelessness about being able to achieve glycemic goals (e.g., in-range HbA1c, avoiding serious complications) [76].

Taken together, the experiences of youth with T1D, including loneliness and social disconnection, burdensomeness related to the demands of T1D management, high cost of treatment and required accommodations, the increased rate of other mental and behavioral health conditions, increased experiences with pain, and increased access to potentially lethal medication, may contribute to the increased risk of suicide-related behaviors seen among this population (see Fig. 1).

\section{Preventive Interventions for Suicide-Related Behaviors Among Youth and Young Adults}

The past decade has seen substantial advances in the empirical evidence-based for suicide prevention in the general population [77-80]. A range of preventive interventions now exist with empirical support for their efficacy, including education-based universal prevention programs (e.g., Question, Persuade, Refer), brief safety-focused interventions (e.g., means safety interventions, safety planning, crisis response planning), and psychosocial interventions (e.g., The Collaborative Assessment and Management of Suicidality, dialectical behavior therapy, cognitive behavioral therapy for suicide prevention) [81-91]. There is also emerging evidence for the use of pharmacological treatment for suicide risk, via the use of single-dose intravenous ketamine, though large-scale clinical trials are needed before pharmacological treatment can be widely implemented [92-94].

Perhaps most critical for use within medical settings, such as diabetes care clinics, are brief safety-focused interventions, including means safety and safety planning interventions. Means safety refers to interventions that involve temporarily restricting an individual's access to items that may be used for suicide, in order to deny or delay access to those items in the event of a suicidal crisis. As suicidal crises are typically brief, with rapid onset and rapid de-escalation $[95,96]$, reducing access to lethal methods can prevent suicidal behavior long enough for de-escalation to occur. In the general population, use of means safety interventions is associated with changes in safety-related behaviors and increased safe storage of firearms and medications [97-101]. 


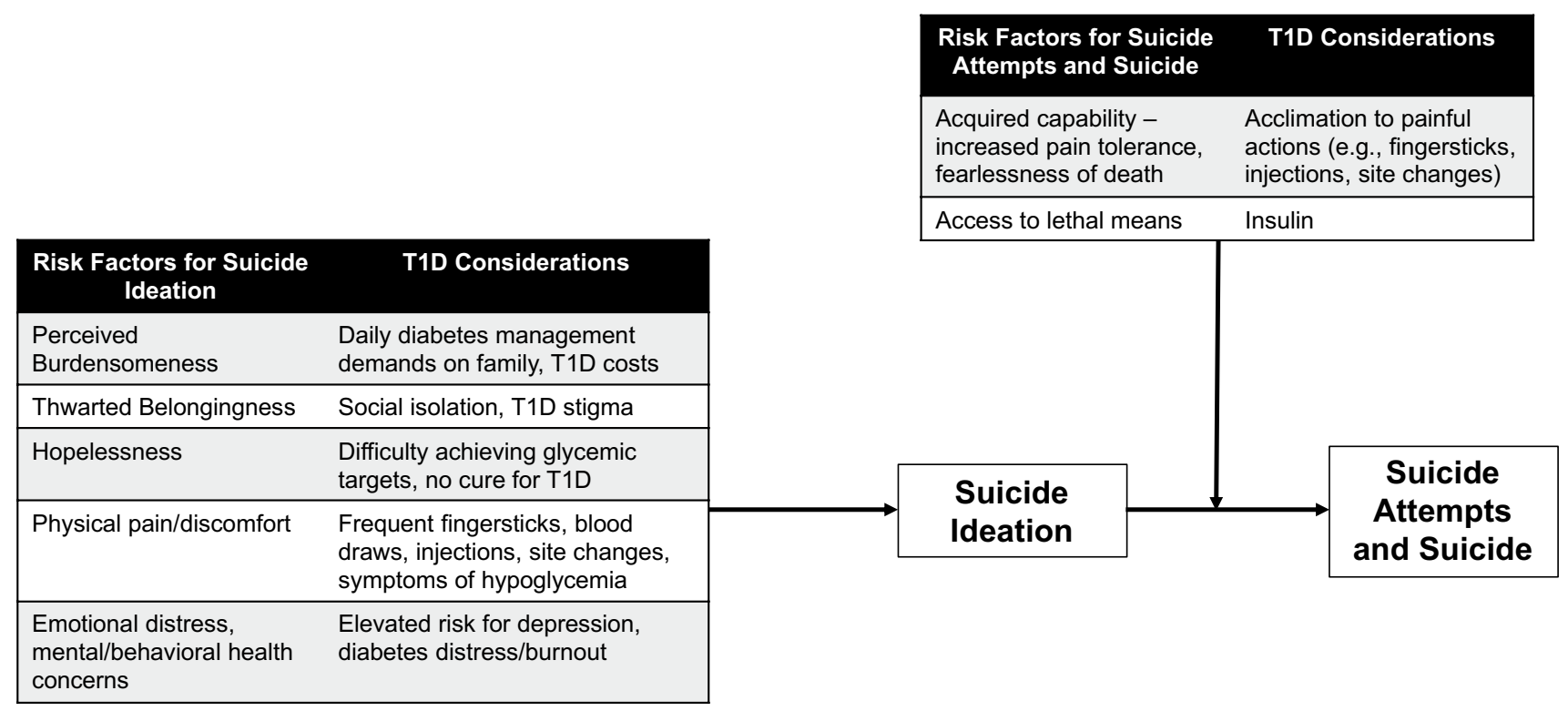

Fig. 1 Hypothesized associations between type 1 diabetes and suicide risk among youth and young adults within an ideation-to-action framework

Counseling on Access to Lethal Means (CALM), a brief means safety intervention, includes providing education to individuals at elevated risk for suicide (including the parents of at-risk youth) regarding the importance of safe storage of lethal means, an explanation of why safe storage is important, and helping individuals identify safe storage steps based on their personal risks and environment. Specifically, CALM highlights the safe storage of firearms, as firearm suicides account for $50 \%$ of all suicide deaths in the USA, and medications, as intentional overdose is the most common method of suicide attempt [102, 103]. CALM can be implemented in as little as $10 \mathrm{~min}$, making it particularly useful in fast-paced medical settings or where provider time and resources are limited, such as emergency departments or pediatric primary care clinics. With brief training, CALM can be implemented by a range of providers, and is frequently administered by nursing staff or discharge counselors [100, 104].

Suicide safety planning is a preventive intervention designed to assist individuals who experience suicidal ideation in maintaining safety during a suicidal crisis [105]. Safety planning is frequently included as an element in cognitive-behavioral interventions for suicide prevention or may be used as a standalone intervention [88]. Safety planning is an interactive process in which a provider and client identify a series of action steps to be taken in the event the client experiences suicidal ideation, arranged in order of increasing response intensity. When conducted with youth, parents may be included in the development of a safety plan or the plan may be shared with parents upon completion. Perhaps the most widely adopted, manualized version of safety planning, the Safety Planning Intervention, includes identifying (a) warning signs or triggers that typically precede a suicidal crisis, (b) internal coping strategies (e.g., distracting activities), (c) social contacts or locations that may provide distraction, (d) supportive contacts who can provide assistance in a crisis, (e) emergency resources (e.g., therapist phone numbers, hotlines, hospital emergency room locations), and (f) limiting access to lethal means of suicide (i.e., safety planning includes a means safety intervention) [105]. Multiple studies now demonstrate the efficacy of safety planning as a standalone, brief intervention [106-108, 109•, 110]. Of note, safety planning is distinct from so-called no-suicide contracts, in which youth sign a paper agreeing not to engage in suicidal behavior. No-suicide contracts are generally not recommended, as they have not been found to be efficacious and raise a number of clinical and ethical concerns [111, 112]. In contrast, safety planning is recommended as a part of routine clinical care for individuals at risk for suiciderelated behaviors $[21,113]$. In practice, suicide safety planning can be completed in approximately 30-40 min. While more time-intensive than a simple means safety intervention, safety planning is brief enough to be utilized in a range of medical settings and is frequently implemented in the context of social work or psychology consult services.

In addition to brief safety-focused interventions, youth identified as at-risk for suicide-related behaviors in medical settings can be referred to outpatient mental or behavioral healthcare providers for ongoing therapy. Suicide risk is a transdiagnostic phenomenon and may be present in the context of a range of other co-occurring mental and behavioral health diagnoses. As a result, outpatient therapy may target 
suicide-related behaviors specifically, such as in the Collaborative Assessment and Management of Suicidality (CAMSCare) [84], or may target other relevant concerns, such as depression, anxiety, or substance abuse issues. Finally, when youth are at imminent risk for engaging in suicidal behavior, inpatient psychiatric hospitalization may be necessary to ensure youth remain safe until a crisis has de-escalated and they may be safely discharged to outpatient therapy.

None of these interventions has been evaluated specifically in youth with T1D. Given that youth with T1D typically have access to insulin for self-management of their blood glucose and that insulin overdose is a serious health risk, special considerations are needed for suicide prevention in this population. Removing access to insulin may be problematic, particularly for youth and young adults who need to complete diabetes management tasks at school or work and in other settings outside of parent/adult oversight. Consequently, providers may need to adapt existing interventions to better address the needs of this population. For example, it may be necessary in some cases for parents or other adults (e.g., school nurses) to temporarily manage all insulin access and administration for youth and young adults with T1D at elevated risk of suicide. For individuals with suicidal ideation who use insulin pumps, switching to insulin injections may be considered, as a means to limit youth's insulin access. However, these approaches may severely limit the youth's independence and social engagement and youth may perceive these changes as a punishment for their suicidal ideation. Given the complexity and effort involved in adult management of youth's insulin and to avoid punishing youth for opening up about suicidal ideation, these options should be taken only after careful consideration of alternative solutions. When youth have not considered insulin as a method of suicide attempt and have sufficient coping skills for managing suicidal ideation, it may be appropriate for them to continue to have access to insulin, with or without additional adult oversight. Decisions regarding insulin access and pump use should be informed by youth, parents, the medical team, and a behavioral health professional with experience in suicide risk assessment and management. Given the complexity of issues such as the need to possess potentially dangerous quantities of insulin, preventing suicide in youth with T1D must also emphasize preventing suicidal thoughts altogether, through early prevention and care for behavioral health concerns.

\section{Recommendations for Providers}

There are several practical steps for advancing suicide prevention among youth and young adults with T1D in hospital, outpatient, and primary care settings. Solutions range from simple, low-cost efforts like enhancing provider education and training with respect to suicide prevention, to more complex efforts to increase the availability of integrated behavioral health services within diabetes specialty care clinics.

\section{Enhance Staff Training in Recognizing and Responding to Suicide Risk}

Training medical and administrative staff in diabetes care settings to recognize warning signs of suicide or to respond to youth in crisis can help increase awareness and destigmatize the topic of suicide and suicide prevention. Education can help dispel myths about suicide, such as the concern that asking about suicide results in increased suicidal ideation or can "put the idea in someone's head," a concern which has been repeatedly contradicted in empirical research [114-116]. Recommended resources for training in recognizing risk factors and warning signs of suicide include the Question, Persuade, Refer program [87, 117, 118], SafeTALK [119], At Risk in Primary Care [120], or Recognizing and Responding to Suicide Risk in Primary Care [121].

\section{Implement Routine Screening and Brief Interventions for Suicide Prevention}

Routine screening in diabetes clinics for suicidal ideation can help detect youth at risk, identify needed safety interventions, and direct them toward appropriate behavioral health services. Furthermore, making mental health and safety screening a routine part of medical care may help reduce stigma and increase acceptability of speaking about these topics-youth and parents may feel less "singled out" if they are informed that everyone is routinely screened, and screening may make it easier for medical providers to introduce these topics during appointments.

There are a number of suicide screening tools available, such as the C-SSRS $[122,123]$ or the Ask Suicide Screening Questions toolkit [124, 125]. Data show that routine screening of suicidal ideation in diabetes clinics is feasible within routine T1D care appointments [73]. Of course, if a diabetes clinic institutes a suicidality screening effort, it is critical to have a protocol in place, including trained staff, to score and interpret screeners and then implement more thorough suicide risk assessments and brief interventions (e.g., suicide safety planning, CALM) with youth identified as at-risk. The ZeroSuicide institute offers an array of resources for providers looking to implement suicide prevention in healthcare settings [126••]. Based on our clinical experiences, development of clear protocols and workflows with suggested language for introducing suicide risk screens and interacting with youth and families can help to facilitate smooth implementation of suicide risk screening and intervention in healthcare settings. Reviewing protocols and workflows with the entire medical and support staff, ensuring roles and 
responsibilities are clear, and ensuring all procedures and policies are accurately documented may also help to reduce staff concerns about implementing suicide prevention efforts in healthcare settings such as a diabetes clinic. Clinics may choose to have medical professionals, such as medical assistants or nurses, complete screenings as part of the routine checking-in process to further normalize these assessments, with follow-up from physicians or social workers as needed for at-risk individuals.

\section{Adopt an Integrated Care Model}

Behavioral healthcare providers often have experience with assessing and managing suicide-related behaviors, as well as in supporting families as they address complex issues. As such, providing co-located, integrated behavioral health services or a behavioral health consultation service may be a valuable resource for supporting youth and families with cooccurring medical and behavioral health concerns, such as suicidal ideation. Integrated care also ensures that all members of the treatment team have access to a shared medical record and can easily and openly communicate concerns as they arise, thereby improving the quality care for youth with T1D. For youth with T1D, behavioral healthcare providers who are familiar with T1D basics and the behavioral, psychological, and family issues related to T1D management may be particularly well suited to address suicidality in this population.

\section{Enhance "Upstream" Prevention Efforts}

Given the complexity of managing suicide-related behaviors among youth with T1D, it is imperative that providers place an increased emphasis on identifying and addressing social and emotional difficulties before suicidal ideation develops. That is, by addressing risk factors for suicide early, it may be possible to prevent development of suicidal ideation. As such, providers should be cognizant of suicide risk factors and take steps to address behavioral health difficulties early. Expressions of perceived burdensomeness or thwarted belongingness, depressive symptoms, anxiety, high levels of stress, or poor social connection (e.g., few friends) may be met with referrals for evaluation or supports by behavioral health providers, ideally with expertise in T1D. Responding to a range of behavioral health concerns is critical to suicide prevention. From a psychiatric standpoint, suicide is a transdiagnostic phenomenon and may occur in the context of a wide range of psychiatric conditions. As such, referring youth to behavioral healthcare for depression, anxiety, substance misuse, or other concerns can contribute to suicide prevention. However, it is important to note that access to behavioral healthcare, both for psychiatric medication and psychosocial therapy, may be limited based on location or insurance status, and substantial waitlists are common.

\section{Reducing Diabetes Distress}

From a clinical diabetes management standpoint, elevated diabetes distress is associated with lower quality of life, lower engagement in diabetes self-management behaviors, suboptimal glycemic outcomes, and behavioral health issues [127, 128], all of which can contribute to suicidal ideation. Using diabetes management devices (e.g., continuous glucose monitors, insulin pumps, sensor-augmented insulin pumps) may reduce everyday diabetes burdens and increase quality of life in youth with T1D and parents [129-131]. Therefore, talking to youth and their parents to identify the most stressful parts of diabetes and considering a transition to advanced diabetes technologies if appropriate may help to reduce perceptions of burdensomeness.

\section{Educate and Support Families}

Educating parents about risk factors and warning signs for suicide can help ensure that parents recognize and address warning signs should they arise. Furthermore, education about mental/behavioral health in general, and specifically about ways to support youth and minimize risk factors for suicide, helps to leverage family members in supporting the mental and behavioral health of youth with T1D. The American Academy of Pediatrics also recommends routine screening for depression during well child visits beginning at age 12 [132] and routine screening can also help to normalize behavioral health topics. Normalization of diabetes distress as a common experience among youth and young adults with T1D may help reduce stigma around this topic. Providing education and behavioral support about the importance of maintaining collaborative family relationships around T1D management, encouraging strong social relationships with peers with T1D and others, and recognizing and managing stress (diabetes distress and other sources of stress) can all help youth with T1D reduce their risk for serious mental health concerns including suicidality. This education about the close relationship between chronic health conditions, including T1D, and psychological concerns is intended to help youth and their parents consider the broad range of issues relevant to good health and high quality of life.

\section{Recommendations for Future Research}

The extant literature on the co-occurrence of T1D and suicide-related behaviors among youth is relatively sparse. Additional research is needed to explore unique risk and protective factors associated with suicide-related behaviors 
among this population, as well as factors or experiences that might contribute to elevated rates of thwarted belongingness, perceived burdensomeness, hopelessness, or the acquired capability for suicide. Researchers should develop and evaluate preventive interventions for use among youth with T1D, including any adaptations or modifications that may improve their efficacy or effectiveness in this population. In addition to adapting suicide prevention-focused interventions to be appropriate for youth and young adults with T1D, it may be valuable to develop and evaluate broad behavioral interventions for youth and young adults with T1D that incorporate aspects of suicide prevention. For example, given the increased risk for suicidality in adolescence and early adulthood and the common gaps in care during the transfer between pediatric and adult healthcare settings, interventions targeting diabetes transition may benefit from monitoring suicidality and integrating suicide prevention strategies. Resilience-promotion interventions for youth with T1D that target mood symptoms and diabetes distress concerns [129-131] may be particularly well-suited to address suicide risk factors and prevention. Given the complexity of management of T1D and the potentially lethal nature of insulin overdose, future research should address T1D treatment challenges when youth are experiencing suicidal ideation. For example, technology solutions that integrate remote monitoring functions with other software (e.g., password dual authentication) could be developed to require secondary authorization for insulin pumps to administer insulin dose. In that manner, a caregiver could closely monitor insulin use for youth at risk of suicide without removing their use of a pump or reducing their ability to engage in activities away from home. Alternatively, future research might examine for which patients insulin restriction is necessary (e.g., identifying which factors predict the use of insulin as a means for suicidal behavior).

\section{Summary and Conclusions}

Despite the elevated prevalence of suicide-related thoughts and behaviors in youth and young adults with T1D coupled with the easy access to potentially life-threatening means (i.e., insulin), there has been relatively little research focused on the association between pediatric diabetes and suiciderelated thoughts and behaviors. In the context of current theories and etiology of suicidal behavior, there are many unique biopsychosocial factors of T1D care and management—such as belongingness, burdensomeness, and access to insulin - that contribute to the elevated suicide risk in this population. Theories of suicidal behaviors developed in other populations can serve as a guide to support diabetes care providers in understanding the interplay between the challenges of living with and managing pediatric diabetes and suicide risk within their patients. Evidence-based interventions for suicidality emphasize the importance of reducing suicidal youths' access to potentially lethal means for suicidal behavior, though there are unique T1D-related treatment challenges to implementing these approaches. Means safety and safety planning interventions should integrate diabetes-specific problem-solving and safety planning, specifically discussions about the dangers of inaccurate insulin dosing and/or overdose with youth, their parents, and their diabetes care providers. Suggestions for suicide prevention for this population would benefit from focusing on provider psychoeducation on the risk factors and warning signs, routine universal risk screening within the context of ambulatory T1D care, and brief targeted early youth and family interventions aimed at prevention of suicide behaviors specific to diabetes management. By way of multidisciplinary care coordination, referrals to pediatric health psychology providers serve as an alternative method to screening and intervention for youth with type 1 diabetes at highest risk for suicidal behaviors and provide inroads for the delivery of timely, effective, and lifesaving care.

Data Availability Not applicable.

Code Availability Not applicable.

\section{Declarations}

Ethics Approval Not applicable.

Consent to Participate Not applicable.

Consent for Publication Not applicable.

Conflict of Interest The authors declare no competing interests.

\section{References}

Papers of particular interest, published recently, have been highlighted as:

- Of importance

$\bullet$ Of major importance

1. Centers for Disease Control and Prevention. WISQARS (Webbased Injury Statistics Query and Reporting System). 2021. https://www.cdc.gov/injury/wisqars/index.html. Accessed 14 Apr 2021.

2. Centers for Disease Control and Prevention. Youth risk behavior surveillance system. 2021. https://www.cdc.gov/healthyyouth/ data/yrbs/index.htm. Accessed 14 Apr 2021.

3. Hill RM, Rufino K, Kurian S, Saxena J, Saxena K, Williams L. Suicide ideation and attempts in a pediatric 
emergency department before and during COVID-19. Pediatrics. 2021;147(3):e2020029280.

4. Barnes AJ, Eisenberg ME, Resnick MD. Suicide and self-injury among children and youth with chronic health conditions. Pediatrics. 2010;125:889-95.

5. Erickson JD, Patterson JM, Wall M, Neumark-Sztainer D. Risk behaviors and emotional well-being in youth with chronic health conditions. Children's Health Care. 2005;34:181-92.

6. Butwicka A, Frisén L, Almqvist C, Zethelius B, Lichtenstein P. Risks of Psychiatric disorders and suicide attempts in children and adolescents with type 1 diabetes: a population-based cohort study. Diabetes Care. 2015;38:453-9.

7. Robinson ME, Simard M, Larocque I, Shah J, Nakhla M, Rahme E. Risk of psychiatric disorders and suicide attempts in emerging adults with diabetes. Diabetes Care. 2020;43:484-6.

8. Buchberger B, Huppertz H, Krabbe L, Lux B, Mattivi JT, Siafarikas A. Symptoms of depression and anxiety in youth with type 1 diabetes: a systematic review and meta-analysis. Psychoneuroendocrinology. 2016;70:70-84.

9. Hilliard ME, Herzer M, Dolan LM, Hood KK. Psychological screening in adolescents with type 1 diabetes predicts outcomes one year later. Diabetes Res and Clin Pract. 2011;94:39-44.

10. Martinez K, Frazer SF, Dempster M, Hamill A, Fleming H, McCorry NK. Psychological factors associated with diabetes self-management among adolescents with type 1 diabetes: a systematic review. J Health Psychol. 2018;23:1749-65.

11. McGill DE, Volkening LK, Pober DM, Muir AB, Young-Hyman DL, Laffel LM. Depressive symptoms at critical times in youth with type 1 diabetes: following type 1 diabetes diagnosis and insulin pump initiation. J Adolesc Health. 2018;62:219-25.

12. Barry-Menkhaus SA, Stoner AM, MacGregor KL, Soyka LA. Special considerations in the systematic psychosocial screening of youth with type 1 diabetes. J Pediatr Psychol. 2021;45:299-310.

13. Marker AM, Patton SR, McDonough RJ, Feingold H, Simon L, Clements MA. Implementing clinic-wide depression screening for pediatric diabetes: an initiative to improve healthcare processes. Pediatr Diabetes. 2019;20:964-73.

14. Corathers S, Mara CA, Chundi PK, Kichler JC. Depression screening of adolescents with diabetes: 5-years of implementation and outcomes. J American Academy of Child and Adolesc Psychiatry. 2019;58:628-32.

15. Iturralde E, Adams RN, Barley RC, Bensen R, Christofferson M, Hanes SJ, et al. Implementation of depression screening and global health assessment in pediatric subspecialty clinics. J Adolesc Health. 2017;61:591-8.

16. Corathers SD, Kichler J, Jones NHY, Houchen A, Jolly M, Morwessel N, et al. Improving depression screening for adolescents with type 1 diabetes. Pediatrics. 2013;132:e1395-402.

17. Kichler J, Harris M, Weissberg-Benchell J. Contemporary roles of the pediatric psychologist in diabetes care. Curr Diabetes Reviews. 2015;11:210-21.

18. Hilliard ME, De Wit M, Wasserman RM, Butler AM, Evans M, Weissberg-Benchell J, et al. Screening and support for emotional burdens of youth with type 1 diabetes: strategies for diabetes care providers. Pediatr Diabetes. 2018;19:534-43.

19. American Diabetes Association. Children and adolescents: standards of medical care in diabetes-2021. Diabetes Care. 2021;44:S180-99.

20. Shain B. Suicide and suicide attempts in adolescents. Pediatrics. 2016;138:e20161420

21. The Joint Commission. Detecting and treating suicide ideation in all settings. http://www.jointcommission.org/assets/1/18/SEA_ 56_Suicide.pdf. Accessed 14 Apr 2021.

22. Roaten K, Johnson C, Genzel R, Khan F, North CS. Development and implementation of a universal suicide risk screening program in a safety-net hospital system. Jt Comm J Quality and Patient Saf. 2018;44:4-11.

23. Heyland M, Delaney KR, Shattell M. Steps to achieve universal suicide screening in emergency departments: a call to action. J Psychosoc Nurs and Ment Health Services. 2018;56:21-6.

24. Hill RM, Rufino K, Kurian S, Saxena J, Saxena K, Williams L. Suicide ideation and attempts in a pediatric emergency department before and during COVID-19. Pediatrics. 2021;147:2020029280.

25. Fuller-Thomson E, Milinovich JL, Merighi JR. Lifetime prevalence of comorbid mood disorders in a representative sample of Canadians with type 1 diabetes. J Diabetes and Its Complications. 2010;24:297-300.

26. Goldston DB, Kovacs M, Ho VY, Parrone PL, Stiffler L. Suicidal ideation and suicide attempts among youth with insulindependent diabetes mellitus. J American Academy of Child and Adolesc Psychiatry. 1994;33:240-6.

27. Goldston DB, Kelley AE, Reboussin DM, Daniel SS, Smith JA, Schwartz RP, et al. Suicidal ideation and behavior and noncompliance with the medical regimen among diabetic adolescents. J American Academy of Child and Adolesc Psychiatry. 1997;36:1528-36.

28. Petit JM, Goueslard K, Chauvet-Gelinier JC, Bouillet B, Vergès B, Jollant F, et al. Association between hospital admission for ketoacidosis and subsequent suicide attempt in young adults with type 1 diabetes. Diabetologia. 2020;63:1745-52.

$29 \bullet$ Wang B, An X, Shi X, Zhang JA. Suicide risk in patients with diabetes: a systematic review and meta-analysis. Euro J Endo. 2017;177(4):R169-R181. This systematic review and metaanalysis provides the strongest evidence, to date, documenting the association between suicide-related behaviors and diabetes.

30. Pompili M, Forte A, Lester D, Erbuto D, Rovedi F, Innamorati M, et al. Suicide risk in type 1 diabetes mellitus: a systematic review. J Psychosomatic Res. 2014;76:352-60.

31 Kyvik KO, Stenager EN, Green A, Svendsen A. Suicides in men with IDDM. Diabetes Care. 1994;17:210-2.

32. Mühlhauser I, Sawicki P, Blank M, Overmann H, Richter B, Berger M. Reliability of causes of death in persons with type I diabetes. Diabetologia. 2002;45:1490-7.

33. Ahola AJ, Harjutsalo V, Saraheimo M, Forsblom C, Groop PH. Purchase of antidepressant agents by patients with type 1 diabetes is associated with increased mortality rates in women but not in men. Diabetologia. 2012;55:73-9.

34. David Batty G, Kivimaki M, Park IS, Jee SH. Diabetes and raised blood glucose as risk factors for future suicide: cohort study of 1234927 Korean men and women. J Epi and Community Health. 2012;66:650-2.

35. Wibell L, Nyström L, Östman J, Arnqvist H, Blohmé G, Lithner $\mathrm{F}$, et al. Increased mortality in diabetes during the first 10 years of the disease: a population-based study (DISS) in Swedish adults 15-34 years old at diagnosis. J Intern Med. 2001;249:263-70.

36. Orchard TJ. International evaluation of cause-specific mortality and IDDM. Diabetes Care. 1991;14:55-60.

37. World Health Organization. Mental health https://www.who.int/ data/gho/data/themes/mental-health Accessed 14 Apr 2021.

38. Feltbower RG, Bodansky HJ, Patterson CC, Parslow RC, Stephenson CR, Reynolds C, et al. Acute complications and drug misuse are important causes of death for children and young adults with type 1 diabetes: results from the Yorkshire register of diabetes in children and young adults. Diabetes Care. 2008;31:922-6.

39. Patterson CC, Dahlquist G, Harjutsalo V, Joner G, Feltbower RG, Svensson J, et al. Early mortality in EURODIAB 
population-based cohorts of type 1 diabetes diagnosed in childhood since 1989. Diabetologia. 2007;50:2439-42.

40. Dahlquist G, Källén B. Mortality in childhood-onset type 1 diabetes: a population-based study. Diabetes Care. 2005;28:2384-7.

41. Warner DP, McKinney PA, Law GR, Bodansky HJ. Mortality and diabetes from a population based register in Yorkshire 1978-93. Archives of Dis in Child. 1998;78:435-8.

42. Harjutsalo V, Forsblom C, Groop PH. Time trends in mortality 61. in patients with type 1 diabetes: nationwide population based cohort study. Br Med J. 2011;343:d5364.

43. Joner G, Patrick S. The mortality of children with type 1 (insulin-dependent) diabetes mellitus in Norway, 1973-1988. Diabetologia. 1991;34:29-32.

44. Lewinsohn P, Rohde P, Seeley J. Adolescent suicidal ideation and attempts: prevalence, risk factors, and clinical implications. Clin Psychol Sci Pract. 1996;3:25-46.

45. Gould MS, King R, Greenwald S, Fisher P, Schwab-Stone M, Kramer R, Flisher AJ, Goodman S, Canino G, Shaffer D. Psychopathology associated with suicidal ideation and attempts among children and adolescents. J Am Acad Child Adolesc Psychiatry. 1998;37:915-23.

46. Williams LB, Laffel LMB, Hood KK. Education and psychological aspects diabetes-specific family conflict and psychological distress in paediatric type 1 diabetes. Diabet Med. 2009;26:908-14.

47. Van Gampelaere C, Luyckx K, van der Straaten S, Laridaen J, Goethals ER, Casteels K, et al. Families with pediatric type 1 diabetes: a comparison with the general population on child well-being, parental distress, and parenting behavior. Pediatr Diabetes. 2020;21:395-408.

48. Borus JS, Laffel L. Adherence challenges in the management of type 1 diabetes in adolescents: prevention and intervention. Curr Opin in Pediatr. 2010;22:405-11.

49. Rechenberg K, Whittemore R, Holland M, Grey M. General and diabetes-specific stress in adolescents with type 1 diabetes. Diabetes Res and Clin Pract. 2017;130:1-8.

50. Peña JB, Kuhlberg JA, Zayas LH, Baumann AA, Gulbas L, Hausmann-Stabile C, et al. Familism, family environment, and suicide attempts among Latina youth. Suicide and Life-Threatening Behav. 2011;41:330-41.

51. Walsh E, Eggert LL. Suicide risk and protective factors among youth experiencing school difficulties: feature article. Int J Ment Health Nurs. 2007;16:349-59.

52. King RA, Schwab-Stone M, Flisher AJ, Greenwald S, Kramer RA, Goodman SH, et al. Psychosocial and risk behavior correlates of youth suicide attempts and suicidal ideation. J Am Acad of Child and Adolesc Psychiatry. 2001;40:837-46.

53. Deville DC, Whalen D, Breslin FJ, Morris AS, Khalsa SS, Paulus MP, et al. Prevalence and family-related factors associated with suicidal ideation, suicide attempts, and self-injury in children aged 9 to 10 years. JAMA Netw Open. 2020;3:e1920956.

54. Fergusson DM, Beautrais AL, Horwood LJ. Vulnerability and resiliency to suicidal behaviours in young people. Psychological Med. 2003;33:61-73.

55. Hill RM, Pettit JW, Green KL, Morgan ST, Schatte DJ. Precipitating events in adolescent suicidal crises: exploring stress-reactive and nonreactive risk profiles. Suicide and Life-Threatening Behav. 2012;42:11-21.

56. Buitron V, Hill RM, Pettit JW, Green KL, Hatkevich C, Sharp C. Interpersonal stress and suicidal ideation in adolescence: an indirect association through perceived burdensomeness toward others. J of Affective Disorders. 2016;190:143-9.

57. Joiner T. Why People Die by Suicide. 1st ed. Cambridge, Mass. Harvard University Press; 2019.
58. David Klonsky E, May AM. The three-step theory (3ST): a new theory of suicide rooted in the "ideation-to-action" framework. Int J Cog Therapy. 2015;8:114-29.

59. Van Orden KA, Witte TK, Cukrowicz KC, Braithwaite SR, Selby EA, Joiner TE. The interpersonal theory of suicide. Psychological Rev. 2010;117:575-600.

60. O'Connor RC, Kirtley OJ. The integrated motivational-volitional model of suicidal behavior. Philos R Soc Lond B Biol Sci. 2018;373(1754):20170268.

61. Stewart SM, Eaddy M, Horton SE, Hughes J, Kennard B. The validity of the interpersonal theory of suicide in adolescence: a review. J Clin Child and Adoles Psychol. 2017;46:437-49.

62. Chu C, Buchman-Schmitt JM, Stanley IH, Hom MA, Tucker RP, Hagan CR, et al. The interpersonal theory of suicide: a systematic review and meta-analysis of a decade of cross-national research. Psychol Bull. 2017;143:1313-45.

63. Hill RM, Pettit JW. Perceived burdensomeness and suiciderelated behaviors in clinical samples: current evidence and future directions. J Clinical Psychol. 2014;70:631-43.

64. Pettit JW, Roberts RE, Lewinsohn PM, Seeley JR, Yaroslavsky I. Developmental relations between perceived social support and depressive symptoms through emerging adulthood: blood is thicker than water. J Fam Psychol. 2011;25:127-36.

65. King CA, Merchant CR. Social and interpersonal factors relating to adolescent suicidality: a review of the literature. Arch Suicide Res. 2008;12(3):181-96.

66. Hill RM, Hunt QA, Oosterhoff B, Yeguez CE, Pettit JW. Perceived burdensomeness among adolescents: a mixed-methods analysis of the contexts in which perceptions of burdensomeness occur. J Soc and Clin Psychol. 2019;38:585-604.

67. Boileau $\mathrm{P}$, Aboumrad B, Bougnères P. Recurrent comas due to secret self-administration of insulin in adolescents with type 1 diabetes. Diabetes Care. 2006;29:430-1.

68. Yang W, Dall TM, Beronjia K, Lin J, Semilla AP, Chakrabarti R, et al. Economic costs of diabetes in the U.S. in 2017. Diabetes Care. 2018;41:917-28.

69. Biniek JF, Johnson W. Spending on individuals with type 1 diabetes and the role of rapidly increasing insulin prices. Washington, DC: Health Care Cost Institute; 2019.

70. Adal E, Önal Z, Ersen A, Yalçın K, Önal H, Aydın A. Recognizing the psychosocial aspects of type 1 diabetes in adolescents. $\mathrm{J}$ Clin Res Pediatr Endo. 2015;7:57-62.

71. Monaghan MC, Hilliard ME, Cogen FR, Streisand R. Nighttime caregiving behaviors among parents of young children with Type 1 diabetes: associations with illness characteristics and parent functioning. Fam Syst Health. 2009;27:28-38.

72. Hilliard ME, Monaghan M, Cogen FR, Streisand R. Parent stress and child behaviour among young children with type 1 diabetes. Child Care Health Dev. 2011;37:224-32.

73. Majidi S, O'Donnell HK, Stanek K, Youngkin E, Gomer T, Driscoll KA. Suicide risk assessment in youth and young adults with type 1 diabetes. Diabetes Care. 2020;43:343-8.

74. Pendley JS, Kasmen LJ, Miller DL, Donze J, Swenson C, Reeves G. Peer and family support in children and adolescents with type 1 diabetes. J Pediatr Psychol. 2002;27:429-38.

75. Walker AF, Schatz DA, Johnson C, Silverstein JH, Rohrs HJ. Disparities in social support systems for youths with type 1 diabetes. Clin Diabetes. 2015;33(2):62-9.

76. Karlsson A, Arman M, Wikblad K. Teenagers with type 1 diabetes-a phenomenological study of the transition towards autonomy in self-management. Intl J Nurs Studies. 2008;45:562-70.

77. Calear AL, Christensen H, Freeman A, Fenton K, Busby Grant J, van Spijker B, et al. A systematic review of psychosocial suicide prevention interventions for youth. Eur Child and AdolescPsychiatry. 2016;25:467-82. 
78. Robinson J, Hetrick SE, Martin C. Preventing suicide in young people: systematic review. Australian and New Zealand J Psychiatry. 2011;45:3-26.

79. Gould MS, Greenberg T, Velting DM, Shaffer D. Youth suicide risk and preventive interventions: a review of the past 10 years. J Am Acad Child Adolesc Psychiatry. 2003;42:386-405.

80. Busby DR, Hatkevich C, McGuire TC, King CA. Evidencebased interventions for youth suicide risk. Current Psychiatry Reports: Springer; 2020.

81. Pistorello J, Jobes DA, Gallop R, Compton SN, Locey NS, $\mathrm{Au}$ JS, et al. A randomized controlled trial of the Collaborative Assessment and Management of Suicidality (CAMS) versus Treatment as Usual (TAU) for suicidal college students. Archives of Suicide Research: Routledge; 2020.

82. Jobes DA, Lento R, Brazaitis K. An evidence-based clinical approach to suicide prevention in the department of defense: the Collaborative Assessment and Management of Suicidality (CAMS). Mil Psychol. 2012;24:604-23.

83. Jobes DA, Anne Comtois K, Brenner LA, Gutierrez PM. Clinical trial feasibility studies of the collaborative assessment and management of suicidality. International Handbook of Suicide Prevention: Research, Policy and Practice. Wiley Blackwell. 2011;383-400.

84. Jobes DA. The collaborative assessment and management of suicidality (cams): an evolving evidence-based clinical approach to suicidal risk. Suicide and Life-Threatening Behavior. 2012;42:640-53.

85. Mitchell SL, Kader M, Darrow SA, Haggerty MZ, Keating NL. Evaluating Question, Persuade, Refer (QPR) suicide prevention training in a college setting. J Coll Stud Psychother. 2013;27:138-48.

86. Tompkins TL, Witt J, Abraibesh N. Does a gatekeeper suicide prevention program work in a school setting? Evaluating training outcome and moderators of effectiveness. Suicide and LifeThreatening Behavior Wiley. 2010;40:506-15.

87. Quinnett P. QPR Gatekeeper Training for Suicide Prevention: The Model, Theory and Research. 2012;1-54. https://www.resea rchgate.net/publication/254002929_QPR_Gatekeeper_Training_ for_Suicide_Prevention_The_Model_Rationale_and_Theory. Accessed 14 Apr 2021.

88. Stanley B, Brown G, Brent DA, Wells K, Poling K, Curry J, et al. Cognitive-behavioral therapy for suicide prevention (cbtsp): treatment model, feasibility, and acceptability. J Am Acad of Child and Adolesc Psychiatry. 2009;48:1005-13.

89. Miller AL, Rathus JH, Linehan MN, Wetzler S, Leigh E. Dialectical behavior therapy adapted for suicidal adolescents. J Prac Psychiatry and Behav Health. 1997;3:78-86.

90. McCauley E, Berk MS, Asarnow JR, Adrian M, Cohen J, Korslund K, et al. Efficacy of dialectical behavior therapy for adolescents at high risk for suicide a randomized clinical trial. JAMA Psychiat. 2018;75:777-85.

91. DeCou CR, Comtois KA, Landes SJ. Dialectical behavior therapy is effective for the treatment of suicidal behavior: a metaanalysis. Behav Ther. 2019;50:60-72.

92. Wilkinson ST, Ballard ED, Bloch MH, Mathew SJ, Murrough JW, Feder A, et al. The effect of a single dose of intravenous ketamine on suicidal ideation: a systematic review and individual participant data meta-analysis. Am J Psychiatry. 2018;175:150-8.

93. Murrough JW, Soleimani L, Price R, Murrough JW, Soleimani $\mathrm{L}$, DeWilde KE, et al. Ketamine for rapid reduction of suicidal ideation: a randomized controlled trial Specificity and Validity of Oxidative Stress Model of Chronic Fatigue Syndrome View project Ketamine for rapid reduction of suicidal ideation: a randomized controlled trial. Psychol Med. 2015;45:3571-80.
94. Ballard ED, Ionescu DF, Vande Voort JL, Niciu MJ, Richards EM, Luckenbaugh DA, et al. Improvement in suicidal ideation after ketamine infusion: relationship to reductions in depression and anxiety. J Psychiatric Res. 2014;58:161-6.

95. Drum DJ, Brownson C, Denmark AB, Smith SE. New data on the nature of suicidal crises in college students: shifting the paradigm. Pro Psychol: Res and Pract. 2009;40:213-22.

96. Deisenhammer EA, Ing CM, Strauss R, Kemmler G, Hinterhuber H, Weiss EM. The duration of the suicidal process: how much time is left for intervention between consideration and accomplishment of a suicide attempt? J Clin Psychiatry. 2009;70:19-24.

97. Sale E, Hendricks M, Weil V, Miller C, Perkins S, McCudden S. Counseling on access to lethal means (calm): an evaluation of a suicide prevention means restriction training program for mental health providers. Comm Ment Health J. 2018;54:293-301.

98. McManus BL, Kruesi MJP, Dontes AE, Defazio CR, Piotrowski JT, Woodward PJ. Child and adolescent suicide attempts: an opportunity for emergency departments to provide injury prevention education. Am J Emerg Med. 1997;15:357-60.

99. Betz ME, Miller M, Barber C, Beaty B, Miller I, Camargo CA, et al. Lethal means access and assessment among suicidal emergency department patients. Depress Anxiety. 2016;33:502-11.

100. Runyan CW, Becker A, Brandspigel S, Barber C, Trudeau A, Novins D. Lethal means counseling for parents of youth seeking emergency care for suicidality. Western J Emerg Med. 2016;17:8-14.

101. Bryan CJ, Stone SL, Rudd MD. A practical, evidence-based approach for means-restriction counseling with suicidal patients. Pro Psychol: Res and Pract. 2011;42:339-46.

102. Sale E, Hendricks M, Weil V, Miller C, Perkins S, McCudden S. Counseling on Access to Lethal Means (CALM): an evaluation of a suicide prevention means restriction training program for mental health providers. Community Ment Health J. 2018;54:293-301.

103. Drapeau CW, McIntosh JL. USA Suicide: 2019 official final data. Washington, DC: American Association of Suicidology. 2020. https://suicidology.org/wp-content/uploads/2021/01/ 2019datapgsv2b.pdf. Accessed 14 Apr 2021.

104. Betz ME, Brooks-Russell A, Brandspigel S, Novins DK, Tung GJ, Runyan C. Counseling suicidal patients about access to lethal means: attitudes of emergency nurse leaders. J Emerg Med Nurs. 2018;44:499-504.

105. Stanley B, Brown GK. Safety planning intervention: a brief intervention to mitigate suicide risk. Cogn and Behav Pract. 2012;19:256-64.

106. Stanley B, Chaudhury SR, Chesin M, Pontoski K, Bush AM, Knox KL, et al. An emergency department intervention and follow-up to reduce suicide risk in the va: acceptability and effectiveness. Psychiatric Serv. 2016;67:680-3.

107. Labouliere CD, Stanley B, Lake AM, Gould MS. Safety planning on crisis lines: feasibility, acceptability, and perceived helpfulness of a brief intervention to mitigate future suicide risk. Suicide and Life-Threatening Behav. 2020;50:29-41.

108. Stanley B, Brown GK, Currier GW, Lyons C, Chesin M, Knox KL. Brief intervention and follow-up for suicidal patients with repeat emergency department visits enhances treatment engagement. Am J Publ Health. 2015;105:1570-2.

109.• Stanley B, Brown GK, Brenner LA, Galfalvy HC, Currier GW, Knox KL, et al. Comparison of the safety planning intervention with follow-up vs usual care of suicidal patients treated in the emergency department. JAMA Psychiatry. 2018;75:894-900. The Suicide Safety Planning Intervention has accrued substantial empirical support as a brief preventive intervention to reduce suicidal behavior. This manuscript describes one 
of the largest, most rigorous studies to date demonstrating the efficacy of suicide safety planning.

110. Hill RM, Dodd CG, Gomez M, Do C, Kaplow JB. The safety planning assistant: feasibility and acceptability of a web-based suicide safety planning tool for at-risk adolescents and their parents. Evidence-Based Pract in Child and Adolesc Ment Health. 2020;5:164-72.

111. David Rudd M, Mandrusiak M, Joiner TE Jr. The case against no-suicide contracts: the commitment to treatment statement as a practice alternative. J Clin Psychol In Session. 2006;62:243-51.

112. Mcmyler C, Pryjmachuk S. Do no-suicide contracts work? J Psychiatric and Ment Health Nurs. 2008;15:512-22.

113. Brown G, Stanley B, Western Interstate Commission for Higher Education, Suicide Prevention Resource Center. Safety planning guide a quick guide for clinicians. Waltham, MA: Education Development Center Inc. 2009. https://www.sprc.org/resou rces-programs/safety-planning-guide-quick-guide-clinicians. Accessed 14 Apr 2021.

114. Blades CA, Stritzke WGK, Page AC, Brown JD. The benefits and risks of asking research participants about suicide: a metaanalysis of the impact of exposure to suicide-related content. Clin Psychol Rev. 2018;64:1-12.

115. Polihronis C, Cloutier P, Kaur J, Skinner R, Cappelli M. What's the harm in asking? A systematic review and meta-analysis on the risks of asking about suicide-related behaviors and self-harm with quality appraisal. Arch Suicide Res. 2020;25:1-23.

116. Dazzi T, Gribble R, Wessely S, Fear NT. Does asking about suicide and related behaviours induce suicidal ideation? What is the evidence? Psychol Med. 2014;44:3361-3.

117. QPR Institute Practical and Proven Suicide Prevention Training QPR Institute (en-US) https://qprinstitute.com/. Accessed 30 Apr 2021.

118. Litteken C, Sale E. Long-Term Effectiveness of the question, persuade, refer (qpr) suicide prevention gatekeeper training program: lessons from missouri. Comm Ment Health J. 2018;54:282-92.

119. SafeTalk Suicide Prevention Course. https://www.livingworks. net/safetalk. Accessed 30 Apr 2021.

120. At-Risk in Primary Care. https://store.kognito.com/products/ primary-care. Accessed 30 Apr 2021.

121. Training \& Accreditation - American Association of Suicidology. https://suicidology.org/training-accreditation/. Accessed 30 Apr 2021

122. Posner K, Brown GK, Stanley B, Brent DA, Yershova KV, Oquendo MA, et al. The Columbia-suicide severity rating scale: initial validity and internal consistency findings from three multisite studies with adolescents and adults. Am J Psychiatry. 2011;168:1266-77.

123. Gipson PY, Agarwala P, Opperman KJ, Horwitz A, King CA. Columbia-suicide severity rating scale: predictive validity with adolescent psychiatric emergency patients. Pediatr Emerg Care. 2015;31:88-94.
124. Horowitz LM, Bridge JA, Teach SJ, Ballard E, Klima J, Rosenstein DL, et al. Ask suicide-screening questions (asq) a brief instrument for the pediatric emergency department. Arch Pediatr Adolesc Med. 2012;166:1170-6.

125. NIMH Ask Suicide-Screening Questions (ASQ) Toolkit. https:// www.nimh.nih.gov/research/research-conducted-at-nimh/asqtoolkit-materials/index.shtml. Accessed 30 Apr 2021.

126.• Zero Suicide. https://zerosuicide.edc.org/. Accessed $30 \mathrm{Apr}$ 2021. The ZeroSuicide initiative and website provide a wide array of resources for healthcare professionals and organizations looking to improve the quality of care that they provide in the area of suicide prevention. Providers who wish to implement policies and procedures to address suicide risk among youth with T1D may benefit from reviewing the systematic approach to suicide prevention outlined by the ZeroSuicide program.

127 Lawrence JM, Yi-Frazier JP, Black MH, Anderson A, Hood KK, Imperatore G, Klingensmith GJ, Naughton M, Mayer-Davis EJ, Seid M. SEARCH for Diabetes in Youth Study Group. Demographic and clinical correlates of diabetes-related quality of life among youth with type 1 diabetes. J Pediatr. 2012;161:201-7.

128. Anderson BJ, Laffel LM, Domenger C, Danne T, Phillip M, Mazza C, Hanas R, Waldron S, Beck RW, Calvi-Gries F, Mathieu C. Factors associated with diabetes-specific healthrelated quality of life in youth with type 1 diabetes: the global teens study. Diabetes Care. 2017;40:1002-9.

129. Jaser SS, Datye K, Morrow T, Sinisterra M, LeStourgeon L, Abadula F, Bell GE, Streisand R. THR1VE! Positive psychology intervention to treat diabetes distress in teens with type 1 diabetes: rationale and trial design. Contemp Clin Trials. 2020;96:106086.

130. Weissberg-Benchell J, Shapiro JB, Bryant FB, Hood KK. Supporting teen problem-solving (steps) 3 year outcomes: preventing diabetes-specific emotional distress and depressive symptoms in adolescents with type 1 diabetes. J Consult Clin Psychol. 2020;88:1019-31.

131. Rosenberg AR, Yi-Frazier JP, Eaton L, Wharton C, Cochrane K, Pihoker C, Baker KS, McCauley E. Promoting resilience in stress management: a pilot study of a novel resilience-promoting intervention for adolescents and young adults with serious illness. J Pediatr Psychol. 2015;40:992-9.

132. American Academy of Pediatrics. Recommendations for preventive pediatric health care. https://downloads.aap.org/AAP/PDF/ periodicity_schedule.pdf Accessed 25 Jun 2021.

Publisher's Note Springer Nature remains neutral with regard to jurisdictional claims in published maps and institutional affiliations. 